Steven N. Harding and Gabriel Picioroaga*

\title{
A generalized Walsh system for arbitrary matrices
}

https://doi.org/10.1515/dema-2019-0006

Received October 14, 2018; accepted December 18, 2018

Abstract: In this paper we study in detail a variation of the orthonormal bases (ONB) of $L^{2}[0,1]$ introduced in [Dutkay D. E., Picioroaga G., Song M. S., Orthonormal bases generated by Cuntz algebras, J. Math. Anal. Appl., 2014, 409(2),1128-1139] by means of representations of the Cuntz algebra $\mathcal{O}_{N}$ on $L^{2}[0,1]$. For $N=2$ one obtains the classic Walsh system which serves as a discrete analog of the Fourier system. We prove that the generalized Walsh system does not always display periodicity, or invertibility, with respect to function multiplication. After characterizing these two properties we also show that the transform implementing the generalized Walsh system is continuous with respect to filter variation. We consider such transforms in the case when the orthogonality conditions in Cuntz relations are removed. We show that these transforms which still recover information (due to remaining parts of the Cuntz relations) are suitable to use for signal compression, similar to the discrete wavelet transform.

Keywords: Cuntz algebras, Walsh basis, Hadamard matrix

\section{Introduction}

It is a well-known fact that the collection of exponential functions $\left\{e^{2 \pi i n x}\right\}_{n \in \mathbb{Z}}$ forms an orthonormal basis (ONB) for the Hilbert space $L^{2}[0,1]$. However, motivated by applications in signal processing, we are interested in working with discrete analogs of the exponential ONB. The collection of Rademacher functions, although orthonormal and piecewise constant with range $\{-1,1\}$, do not form a complete set in $L^{2}[0,1]$. In [2] the Rademacher functions were used to construct an ONB that we call in this paper the classic Walsh system. Many generalizations can be found in the literature, e.g., identifying the Walsh functions as characters over the dyadic group in [3], identifying the Rademacher functions with $N$-adic exponentials in [4], etc.

In this paper we study in more detail the generalized Walsh ONB system found in [1]. We will observe that many interesting results about periodicity, invertibility, transform continuity of these type of functions can actually be obtained in a slightly more general setting, where the system is generated by an arbitrary $N \times M$ matrix.

We begin by presenting a few of the results of [1] regarding how a Cuntz algebra representation generates the construction of the generalized Walsh ONB on $L^{2}[0,1]$. Let $A \in \mathbb{C}^{N \times N}$, the space of $N \times N$ complex-valued matrices, be unitary with first row elements $1 / \sqrt{N}$. We adopt the convention that all indexing starts at zero. Letting $\chi_{A}$ be the characteristic function supported on $A$, we define the filter functions $\left\{m_{i}\right\}_{i=0}^{N-1}$ on $L^{2}[0,1]$ as follows:

$$
m_{i}(x)=\sqrt{N} \sum_{j=0}^{N-1} A_{i, j} \chi_{[j / N,(j+1) / N)}(x) .
$$

Steven N. Harding: Iowa State University, Department of Mathematics, 411 Morrill Road, Ames, IA 50011, U.S.A.; E-mail:sharding@iastate.edu

${ }^{\star}$ Corresponding Author: Gabriel Picioroaga: University of South Dakota, Department of Mathematical Sciences, 414 E. Clark Street, Vermillion, SD 57069, U.S.A.; E-mail: Gabriel.Picioroaga@usd.edu 
Note that $m_{0} \equiv 1$. Letting $r(x)=N x \bmod 1$, we define the operators $\left\{S_{i}\right\}_{i=0}^{N-1}$ on $L^{2}[0,1]$ as follows:

$$
S_{i} f(x)=m_{i}(x)(f \circ r)(x) .
$$

Note that $S_{0} 1=1$.

Theorem 1.1. [1] The operators $\left\{S_{i}\right\}_{i=0}^{N-1}$ form a representation of the Cuntz algebra $\mathcal{O}_{N}$ on $L^{2}[0,1]$, i.e.,

$$
\begin{aligned}
S_{i}^{\star} S_{j} & =\delta_{i, j} I_{L^{2}[0,1]}, \\
\sum_{i=0}^{N-1} S_{i} S_{i}^{\star} & =I_{L^{2}[0,1]} .
\end{aligned}
$$

Theorem 1.2. [1] The family

$$
\mathcal{W}^{A}:=\left\{S_{w_{1}} \ldots S_{w_{n}} 1 \mid n \in \mathbb{Z}_{+}, w_{1}, \ldots, w_{n} \in\{0, \ldots, N-1\}\right\}
$$

is an orthonormal basis for $L^{2}[0,1]$, discarding repetitions generated by the fact that $S_{0} 1=1$. We refer to $\mathcal{W}^{A}$ as the generalized Walsh basis corresponding to the matrix $A$.

One description of the elements of $\mathcal{W}^{A}$ is as follows: Let $n \in \mathbb{Z}_{+}$, and consider its usual decomposition in base $N$,

$$
n=i_{0}+i_{1} N+\ldots+i_{k} N^{k}
$$

where $i_{0}, i_{1}, \ldots, i_{k-1} \in\{0,1, \ldots, N-1\}$. The general Walsh function of index $n$ is then given by

$$
W_{n, A}(x)=m_{i_{0}}(x) m_{i_{1}}(r x) \ldots m_{i_{k}}\left(r^{k} x\right),
$$

where $r^{k}=r \circ r \circ \ldots \circ r$ for $k$ functions.

As the name suggests, the general Walsh ONB transcends the classic Walsh ONB. To be precise, the classic Walsh ONB coincides with the general Walsh ONB corresponding to the matrix

$$
A=\frac{1}{\sqrt{2}}\left(\begin{array}{cc}
1 & 1 \\
1 & -1
\end{array}\right) .
$$

See [5] for applications of the general Walsh ONB to signal processing.

A natural way to extend (1.3) and (1.1) for arbitrary matrices $A \in \mathbb{C}^{N \times M}$ is to mimic the above construction without emphasis on the size of $A$. Specifically we redefine $r(x)=M x \bmod 1$ as well as the filters $\left\{m_{i}\right\}_{i=0}^{N-1}$ as follows:

$$
m_{i}(x)=\sqrt{M} \sum_{j=0}^{M-1} A_{i, j} \chi_{[j / M,(j+1) / M)}(x) .
$$

Further define $W_{n, A}:[0,1] \rightarrow \mathbb{C}$ and $S_{i}: L^{2}[0,1] \rightarrow L^{2}[0,1]$ as in (1.3) and (1.1), respectively, with filters as in (1.4). We will continue to refer to the collection $\left\{W_{n, A}\right\}_{n=0}^{\infty}$ for $A \in \mathbb{C}^{N \times M}$ as general Walsh; however, we note that the general Walsh set does not form an ONB when $N \neq M$. In this paper, we examine properties and applications of these rectangular matrix constructions.

\section{Periodicity and invertibility of the general Walsh set}

Many benefits arise from having various modes of periodicity, e.g., reduction of computational complexity. The periodic nature of the classic Walsh functions, in terms of the dyadic intervals of the unit interval, are 
apparent from the Rademacher function construction. In fact, every classic Walsh function has some mode of periodicity, yet this is not the case for general Walsh functions. In this section, we characterize the periodic structure of the general Walsh set, as well as mention some of their algebraic properties.

The construction of the general Walsh function in (1.3) suggests an intrinsic periodic nature which is closely related to the dimension of the associated matrix. In the figure below, we provide the plots of a few general Walsh functions associated with the following matrix to illustrate this observation:

$$
A=\left(\begin{array}{ccc}
1 / \sqrt{3} & 1 / \sqrt{3} & 1 / \sqrt{3} \\
1 / \sqrt{14} & 2 / \sqrt{14} & -3 / \sqrt{14} \\
5 / \sqrt{42} & -4 / \sqrt{42} & -1 / \sqrt{42}
\end{array}\right) .
$$

Notice that plots (D), (E), and (F) exhibit repeating units, whose units of repetition may be described in terms of plots (A), (B), and (C), respectively. Furthermore, the number of repeating units can be ascertained by the ratio of their indices. For example, the order of the general Walsh function depicted in (E) is $3^{2}$ times the order of its corresponding function depicted in (B); consequently, plot (E) may be described by repeating units attained by compressing plot (B) into the interval $\left[0,1 / 3^{2}\right.$ ) and then extending periodically. See [6] for results pertinent to these observations. Here we present an alternative approach to characterizing periodicity. First, we observe the following lemma.

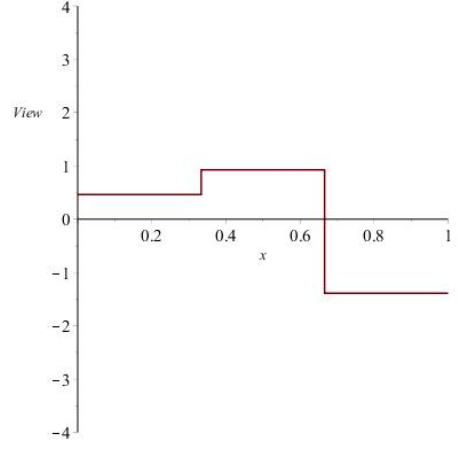

(a) $W_{1, A}(x)$

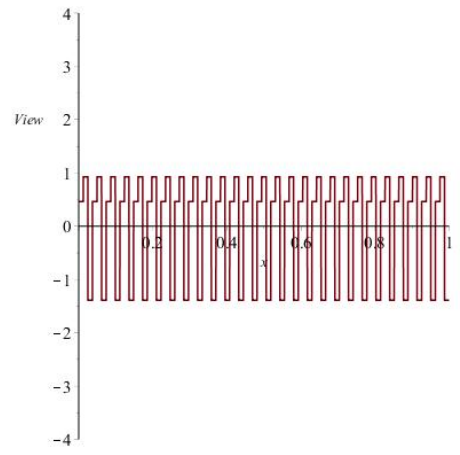

(d) $W_{27, A}(x)$

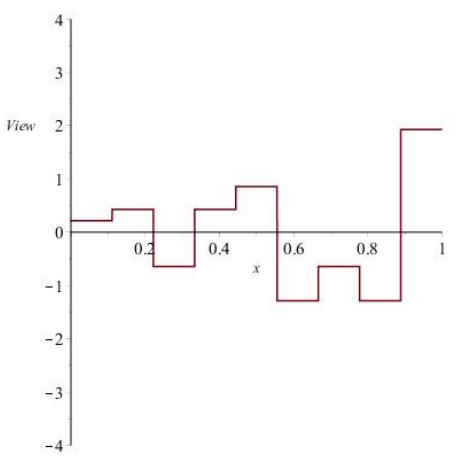

(b) $W_{4, A}(x)$

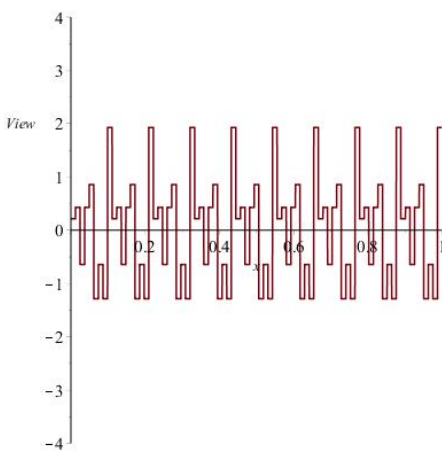

(e) $W_{36, A}(x)$

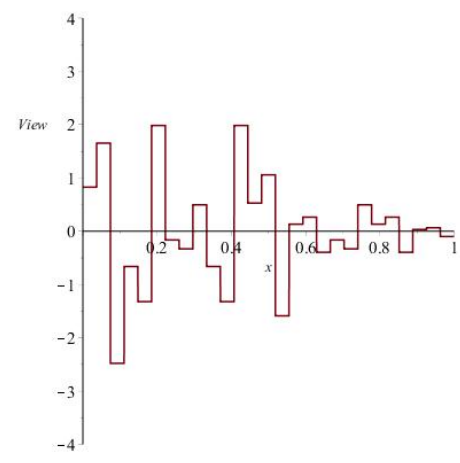

(c) $W_{17, A}(x)$

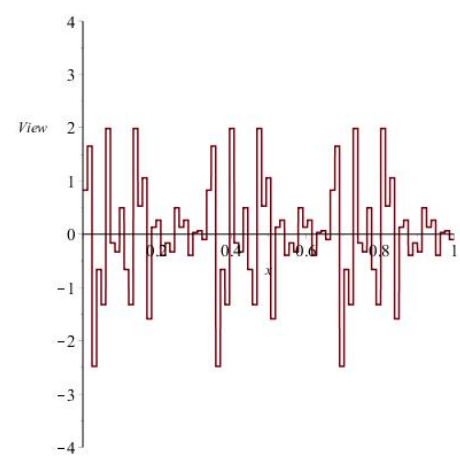

(f) $W_{51, A}(x)$

Figure 1: The plots of six general Walsh functions associated corresponding to (2.1).

Lemma 2.1. Let $A \in \mathbb{C}^{N \times M}$ be with no row consisting entirely of zeros. Let $n \in \mathbb{N}_{0}$, and consider its base- $N$ decomposition (1.2). Then

$$
W_{n, A}(x)=m_{i_{0}, A}(x) W_{\left(n-i_{0}\right) / N, A}(r x)
$$


and, for every $j \in\{0,1, \ldots, M-1\}$, there exists an interval $I_{j} \subset\left[\frac{j}{M}, \frac{j+1}{M}\right)$, such that $W_{\left(n-i_{0}\right) / N, A}(r x) \neq 0$ for $x \in I_{j}$.

Proof. It is straightforward to show the identity, so we omit that part of the proof. Instead, we prove the latter statement. We begin by fixing $j \in\{0,1, \ldots, M-1\}$. Since every row of $A$ contains a nonzero element, let $j_{t} \in\{0,1, \ldots, M-1\}$, such that $A_{i_{t}, j_{t}} \neq 0$ for every $1 \leq t \leq k$. Now consider the chain:

$$
\begin{aligned}
I_{j}:=\left[\frac{j_{k}}{M^{k+1}}, \frac{j_{k}+1}{M^{k+1}}\right) & +\frac{j M^{k-1}+j_{1} M^{k-2}+\ldots+j_{k-1}}{M^{k}} \subset \ldots \\
& \ldots \subset\left[\frac{j_{2}}{M^{3}}, \frac{j_{2}+1}{M^{3}}\right)+\frac{j M+j_{1}}{M^{2}} \subset\left[\frac{j_{1}}{M^{2}}, \frac{j_{1}+1}{M^{2}}\right)+\frac{j}{M} \subset\left[\frac{j}{M}, \frac{j+1}{M}\right) .
\end{aligned}
$$

Then, for $x \in I_{j}$, we have

$$
\begin{aligned}
W_{\left(n-i_{0}\right) / N, A}(r x) & =m_{i_{1}}(r x) m_{i_{2}}\left(r^{2} x\right) \ldots m_{i_{k}}\left(r^{k} x\right) \\
& =A_{i_{1}, j_{1}} A_{i_{2}, j_{2}} \ldots A_{i_{k}, j_{k}} \\
& \neq 0
\end{aligned}
$$

which completes the proof of the lemma.

By the nature of the function $r$, we observe that the function $W_{\left(n-i_{0}\right) / N, A} \circ r$ exhibits periodicity. In particular,

$$
W_{\left(n-i_{0}\right) / N, A}(r(x+j / M))=W_{\left(n-i_{0}\right) / N, A}(r(x)) .
$$

We will regard this property as periodic on the unit interval with the usual notion of periodicity. Then, from Lemma 2.1, we may characterize when a general Walsh function is periodic in terms of the filter $m_{i_{0}}$.

Theorem 2.2. Let $A \in \mathbb{C}^{N \times M}$ be with no row consisting entirely of zeros. $W_{n, A}$ is periodic if and only if $m_{i_{0}}$ is periodic.

Proof. If $m_{i_{0}}$ is periodic, then there exists a positive integer $s<M$, such that $m_{i_{0}}(x)=m_{i_{0}}(x+s / M)$ for $0 \leq x<1-s / M$. From Lemma 2.1, we find that $W_{n, A}$ is periodic because

$$
\begin{aligned}
W_{n, A}(x+s / M) & =m_{i_{0}, A}(x+s / M) W_{\left(n-i_{0}\right) / N, A}(r(x+s / M)) \\
& =m_{i_{0}, A}(x) W_{\left(n-i_{0}\right) / N, A}(r x) \\
& =W_{n, A}(x) .
\end{aligned}
$$

Conversely, if $W_{n, A}$ is periodic, then there exists a positive integer $t<M$, such that $t / M$ is the period of $W_{n, A}$. Then, from the identity in Lemma 2.1, we have

$$
\begin{aligned}
m_{i_{0}}(x+t / M) W_{\left(n-i_{0}\right) / N, A}(r x) & =m_{i_{0}}(x+t / M) W_{\left(n-i_{0}\right) / N, A}(r(x+t / M)) \\
& =W_{n, A}(x+t / M) \\
& =W_{n, A}(x) \\
& =m_{i_{0}}(x) W_{\left(n-i_{0}\right) / N, A}(r x),
\end{aligned}
$$

for $0 \leq x<1-t / M$. Again by Lemma 2.1, for every $0 \leq j \leq M-t-1$, there exists an interval $I_{j} \subset\left[\frac{j}{M}, \frac{j+1}{M}\right)$, such that $W_{\left(n-i_{0}\right) / N, A}(r x) \neq 0$ for $x \in I_{j}$. Hence,

$$
m_{i_{0}}(x+t / M)=m_{i_{0}}(x),
$$

for $x \in I_{j}$. Since $m_{i_{0}}$ is constant on $M$-adic intervals, it follows that $m_{i_{0}}$ is periodic. 
Now we will discuss some algebraic properties of the general Walsh set. Let $\times$ be the operation on $L^{2}[0,1]$ given by $(f \times g)(x)=f(x) \overline{g(x)}$. Although $\times$ is neither binary nor commutative on the general Walsh set, we will investigate right and left invertibility in $\left(\left\{W_{i, A}\right\}_{i=0}^{\infty}, \times\right)$. We will impose the conditions:

(i) No row other than the first row can be a scalar multiple of the all ones vector.

(ii) The Schur product of rows $r_{i}$ and $\overline{r_{j}}, i \neq j$, is not a scalar multiple of the all ones vector.

(iii) The $\ell^{2}$-norm of any row vector is 1 .

Examples of matrices satisfying all three conditions are $N \times M$ submatrices of an $M \times M$ Hadamard or Fourier matrix. However, there is a plethora of examples which are not submatrices of the Fourier or Hadamard that satisfy the requirements above, for example:

$$
A=\left(\begin{array}{ccc}
1 \sqrt{3} & 1 \sqrt{3} & 1 \sqrt{3} \\
0 & 0 & 1
\end{array}\right)
$$

To characterize invertibility under these conditions, we begin with the following lemma.

Lemma 2.3. Let $A \in \mathbb{C}^{N \times M}$ satisfy conditions (i) and (ii). Then $W_{n, A}$ is left or right invertible in $\left(\left\{W_{i, A}\right\}_{i=0}^{\infty}, \times\right)$ if and only if $\left|W_{n, A}(x)\right|=1$.

Proof. The proof is similar for both left and right invertibility. Hence, suppose that $W_{n, A}$ is right invertible in $\left(\left\{W_{i, A}\right\}_{i=0}^{\infty}, \times\right)$. Then there exists $m \in \mathbb{N}_{0}$, such that $W_{n, A} \times W_{m, A}=1$. Consider the base- $N$ decomposition of $n$ and $m$,

$$
\begin{aligned}
& n=i_{0}+i_{1} N+\ldots+i_{k} N^{k} \\
& m=j_{0}+j_{1} N+\ldots+j_{l} N^{l} .
\end{aligned}
$$

We may assume without loss of generality that $k \geq l$ since $W_{n, A} \times W_{m, A}=\overline{W_{m, A} \times W_{n, A}}=1$. However, assume for a contradiction that $k>l$. Note that $i_{k} \neq 0$. Upon regrouping, we have

$$
\left(m_{i_{0}}(x) \overline{m_{j_{0}}(x)}\right)\left(m_{i_{1}}(r x) \overline{m_{j_{1}}(r x)}\right) \ldots\left(m_{i_{l}}\left(r^{l} x\right) \overline{m_{j_{l}}\left(r^{l} x\right)}\right) m_{i_{l+1}}\left(r^{l+1} x\right) \ldots, m_{i_{k}}\left(r^{k} x\right)=1 .
$$

By the proof of Lemma 2.1, we may choose an interval $I$ small enough, such that for all $x \in I$ all terms in the product above except $m_{i_{k}}\left(r^{k} x\right)$ are constant. By i) this would not be possible unless $i_{k}=0$, i.e., the first row in matrix $A$. Hence $k=l$. The same argument can be made to show that $i_{l}=j_{l}, \ldots, i_{0}=j_{0}$ by condition (ii). The converse is straightforward, and this concludes the proof.

Theorem 2.4. Let $A \in \mathbb{C}^{N \times M}$ satisfy conditions (i), (ii) and (iii). Let $n \in \mathbb{N}_{0}$, and consider its base- $N$ decomposition (1.2). Then $W_{n, A}$ is left or right invertible in $\left(\left\{W_{i, A}\right\}_{i=0}^{\infty}, \times\right)$ if and only if

$$
\left|A_{i_{j}, 0}\right|^{2}=\left|A_{i_{j}, 1}\right|^{2}=\ldots=\left|A_{i_{j}, M-1}\right|^{2}=1 / M \text { for } 0 \leq j \leq k .
$$

Proof. Suppose that $W_{n, A}$ is invertible in $\left(\left\{W_{i, A}\right\}_{i=0}^{\infty}, \times\right)$. Consider the product of filter functions

$$
W_{n, A}(x)=m_{i_{0}}(x) m_{i_{1}}(r x) \ldots m_{i_{k-1}}\left(r^{k-1} x\right) m_{i_{k}}\left(r^{k} x\right) .
$$

For $x \in\left[0,1 / M^{k}\right)$, we have $r^{l}(x) \in\left[0,1 / M^{k-l}\right.$ ) when $l \leq k$. In particular, when $l<k$, the inequality $r^{l}(x)<1 / M$ implies that $m_{i_{l}}\left(r^{l} x\right)$ is constant. Thus, on the interval $\left[0,1 / M^{k}\right)$, the product $m_{i_{0}}(x) m_{i_{1}}(r x) \ldots m_{i_{k-1}}\left(r^{k-1} x\right)$ is independent of $x$. Let $b$ be this constant, so we have

$$
\left.W_{n, A}\right|_{\left[0,1 / M^{k}\right)}(x)=\left.b m_{i_{k}}\right|_{\left[0,1 / M^{k}\right)}\left(r^{k} x\right) .
$$

From the definition of the filter function, we have

$$
\left.m_{i_{k}}\right|_{\left[0,1 / M^{k}\right)}\left(r^{k} x\right)=\sqrt{M} \sum_{l=0}^{M-1} A_{i_{k}, l} \chi_{\left[l / M^{k+1},(l+1) / M^{k+1}\right)}(x) .
$$


It follows from this and Lemma 2.3 that

$$
\sqrt{M}\left|b A_{i_{k}, 0}\right|=\sqrt{M}\left|b A_{i_{k}, 1}\right|=\ldots=\sqrt{M}\left|b A_{i_{k}, M-1}\right|=1 .
$$

By summing the square of these terms and observing condition (iii), we find

$$
|b|^{2} M=|b|^{2} M\left(\left|A_{i_{k}, 0}\right|^{2}+\left|A_{i_{k}, 1}\right|^{2}+\ldots+\left|A_{i_{k}, M-1}\right|^{2}\right)=M .
$$

Therefore, $|b|=1$ and consequently $\left|A_{i_{k}, 0}\right|^{2}=\left|A_{i_{k}, 1}\right|^{2}=\ldots=\left|A_{i_{k}, M-1}\right|^{2}=1 / M$. Moreover, this implies $\left|m_{i_{k}}(x)\right|=1$, and we have

$$
\left|W_{n, A}(x)\right|=\left|m_{i_{0}}(x) m_{i_{1}}(r x) \ldots m_{i_{k-1}}\left(r^{k-1} x\right)\right|=1 .
$$

We repeat this argument until we have exhausted all of the $i_{j}$. The converse follows directly from Lemma 2.3.

Corollary 2.5. Let A be a Hadamard matrix. Then every general Walsh function is invertible in $\left(\left\{W_{i, A}\right\}_{i=0}^{\infty}, \times\right)$.

\section{Continuity and error estimates}

In this section, we discuss results pertinent to error approximation analysis much like that of the following theorem from [7].

Theorem 3.1. [7] Let $A \in \mathbb{C}^{N \times N}$ be a unitary matrix with $1 / \sqrt{N}$ for first row entries. If $f$ is constant on the $N$-adic intervals $\left\{\left[k / N^{q},(k+1) / N^{q}\right)\right\}_{k=0}^{N^{q}-1}$, then

$$
f(x)=\sum_{n=0}^{N^{q}-1}\left\langle f, W_{n, A}\right\rangle W_{n, A}(x),
$$

for every $x \in[0,1)$.

A function which is constant on $N$-adic intervals may be treated as an encoding of data and, hence, finds applications in signal processing, e.g., estimating the error in reconstructing a signal $f$ from its frequencies $\left\{\left\langle f, W_{n, A}\right\rangle\right\}$ using an approximated matrix $B$. We will make this explicit next. Let $A, B \in \mathbb{C}^{N \times M}$, and let $q \in \mathbb{Z}_{+}$. Let $P C_{q}$ be the collection of piecewise constant functions $f:[0,1] \rightarrow \mathbb{C}$ of the form

$$
f=\sum_{j=0}^{M^{q}-1} \alpha_{j} \chi_{\left[j / M^{q},(j+1) / M^{q}\right)},
$$

where $\left\{\alpha_{j}\right\}_{j=0}^{M^{q}-1} \subset \mathbb{C}$. We define the analysis operator $\Theta_{A}: P C_{q} \rightarrow \mathbb{C}^{M^{q}}$ by $\Theta_{A} f=\left(\left\langle f, W_{n, A}\right\rangle\right)_{n=0}^{M^{q}-1}$, and the mixed frame operator is then defined by $\Theta_{B}^{\star} \Theta_{A}$, that is

$$
\Theta_{B}^{\star} \Theta_{A} f=\sum_{n=0}^{M^{q}-1}\left\langle f, W_{n, A}\right\rangle W_{n, B} .
$$

When $A=B$ is an $N \times N$ unitary matrix with first row elements $1 / \sqrt{N}$, Theorem 3.1 guarantees the recovery $\Theta_{B}^{\star} \Theta_{A} f=f$. In the context of general $A$ and $B$, we will observe this reconstruction identity, as well as upper estimates on the error operator $I-\Theta_{B}^{\star} \Theta_{A}$ on $P C_{q}$. To that end, let $\theta: P C_{q} \rightarrow \mathbb{C}^{M^{q}}$ be given by $\theta f(j)=f\left((j+1 / 2) / M^{q}\right)$. We will perform our analysis of the error operator in the sequence space through this map. 
We begin by recalling the tensor product. Let $A \in \mathbb{C}^{N_{1} \times M_{1}}$ and $B \in \mathbb{C}^{N_{2} \times M_{2}}$. Recall the tensor product of $A$ with $B$, denoted $A \otimes B$, is defined as follows:

$$
A \otimes B=\left(\begin{array}{cccc}
A_{0,0} B & A_{0,1} B & \ldots & A_{0, M_{1}-1} B \\
A_{1,0} B & A_{1,1} B & \ldots & A_{1, M_{1}-1} B \\
\vdots & \vdots & \ddots & \vdots \\
A_{N_{1}-1,0} B & A_{N_{1}-1,1} B & \ldots & A_{N_{1}-1, M_{1}-1} B
\end{array}\right) .
$$

The following lemma gives a method by which we may compute the general Walsh function. This will be needed in characterizing recovery.

Lemma 3.2. Let $A \in \mathbb{C}^{N \times M}$. Let $n \in\left\{0,1, \ldots, N^{q}-1\right\}$ and $m \in\left\{0,1, \ldots M^{q}-1\right\}$, and consider their respective base decompositions:

$$
\begin{gathered}
n=k_{0}+k_{1} N+\ldots+k_{q-1} N^{q-1} \\
m=i_{0}+i_{1} M+\ldots+i_{q-1} M^{q-1} .
\end{gathered}
$$

Then, in the context of sequences, we have

$$
\left(\theta W_{n, A}\right)(m)=\sqrt{M}^{q} A_{k_{0}, i_{q-1}} A_{k_{1}, i_{q-2}} \ldots A_{k_{q-1}, i_{0}} .
$$

Proof. We evaluate each $\left(\theta m_{k_{s}}\right)\left(r^{s} m\right)$ for $0 \leq s \leq q-1$. Let $A_{s}$ be the vector of length $M^{q-s}$,

$$
A_{s}=\sqrt{M}\left(A_{k_{s}, 0}, \quad \ldots, \quad A_{k_{s}, 0}, \quad A_{k_{s}, 1}, \quad \ldots, \quad A_{k_{s}, 1}, \ldots, A_{k_{s}, M-1}, \ldots, A_{k_{s}, M-1}\right),
$$

with each string, e.g., $A_{k_{s}, 0}, \ldots, A_{k_{s}, 0}$, consisting of $M^{q-s-1}$ elements. Note that $\theta \circ m_{k_{s}} \circ r^{s}$ is the vector $\left(A_{s}\right)^{\oplus M^{s}}$, that is the direct sum $A_{s} \oplus \ldots \oplus A_{s}$ for $M^{s}$-many vectors. Since $M^{q-s}$ divides $i_{q-s} M^{q-s}+\ldots+i_{q-1} M^{q-1}$, we have that $\left(\theta m_{k_{s}}\right)\left(r^{s} m\right)$ is the element of $A_{s}$ whose index is $i_{0}+i_{1} M+\ldots+i_{q-s-1} M^{q-s-1}$. Thus, we find $\left(\theta m_{k_{s}}\right)\left(r^{s} m\right)=$ $\sqrt{M} A_{k_{s}, i_{q-s-1}}$.

To estimate the pointwise error of recovery from (3.1), we will impose the $L^{\infty}$-norm on $P C_{q}$. To emphasize that we are restricting ourselves to the subspace $P C_{q} \subset L^{\infty}[0,1]$, we will denote the norm by $\|\cdot\|_{P C_{q}}$. It is clear that $\|f\|_{P C_{q}}=\|\theta f\|_{\ell^{\infty}\left(\mathbb{C}^{M^{q}}\right)}$. If we let $T$ be an operator on $P C_{q}$ and $S$ be the corresponding matrix representation of $T$, i.e., $\theta(T f)=S(\theta f)$, then

$$
\|T\|_{P C_{q}}=\sup _{\|f\|_{P C_{q}=1}}\|T f\|_{P C_{q}}=\sup _{\|\theta f\|_{\ell^{\infty}\left(\mathbb{C}^{M^{q}}\right)}=1}\|S(\theta f)\|_{\ell^{\infty}\left(\mathbb{C}^{M^{q}}\right)}=\|S\|_{\ell^{\infty}\left(\mathbb{C}^{M^{q}}\right)} .
$$

The next theorem characterizes perfect recovery. Furthermore, its proof may be used to estimate an upper bound on the norm of the error operator on $P C_{q}$ in terms of the matrices $A$ and $B$ that were implemented in its construction.

Theorem 3.3. Let $A, B \in \mathbb{C}^{N \times M}$. We have $I-\Theta_{B}^{\star} \Theta_{A}=0$ if and only if $A^{\star} B=I_{M}$.

Proof. We determine the matrix representation $S$ of the mixed frame operator. Let $\left\{e_{m}\right\}_{m=0}^{M^{q}-1}$ be the canonical ONB of $\mathbb{C}^{M^{q}}$, and let $\delta_{m} \in P C_{q}$ where $e_{m}=\theta \delta_{m}$. Let $m, m^{\prime} \in\left\{0,1, \ldots, M^{q}-1\right\}$, and consider their base- $M$ decomposition:

$$
\begin{aligned}
m & =i_{0}+i_{1} M+\ldots+i_{q-1} M^{q-1} \\
m^{\prime} & =j_{0}+j_{1} M+\ldots+j_{q-1} M^{q-1}
\end{aligned}
$$


From Lemma 3.2, we find

$$
\begin{aligned}
S\left(e_{m}\right)\left(m^{\prime}\right) & =\theta\left[\Theta_{B}^{\star} \Theta_{A} \delta_{m}\right]\left(m^{\prime}\right) \\
& =\sum_{n=0}^{M^{q}-1}\left\langle\delta_{m}, W_{n, A}\right\rangle_{L^{2}[0,1]}\left(\theta W_{n, B}\right)\left(m^{\prime}\right) \\
& =\sum_{n=0}^{M^{q}-1} \frac{\left\langle e_{m}, \theta W_{n, A}\right\rangle_{\ell^{2}\left(\mathbb{C}^{M^{q}}\right)}}{M^{q}}\left(\theta W_{n, B}\right)\left(m^{\prime}\right) \\
& =\frac{1}{M^{q}} \sum_{n=0}^{M^{q}-1} \frac{\left(\theta W_{n, A}\right)(m)}{\left(\theta W_{n, B}\right)\left(m^{\prime}\right)} \\
& =\sum^{N-1} \frac{k_{0}, \ldots, k_{q-1}=0}{A_{k_{0}, i_{q-1}} A_{k_{1}, i_{q-2}} \ldots A_{k_{q-1}, i_{0}}} B_{k_{0}, j_{q-1}} B_{k_{1}, j_{q-2}} \ldots B_{k_{q-1}, j_{0}} \\
& =\left(A^{\star} B\right)_{i_{q-1}, j_{q-1}}\left(A^{\star} B\right)_{i_{q-2}, j_{q-2}} \ldots\left(A^{\star} B\right)_{i_{0}, j_{0}} .
\end{aligned}
$$

Thus the matrix representation of $\Theta_{B}^{\star} \Theta_{A}$ is $\left(\left(A^{\star} B\right)^{T}\right)^{\otimes q}$, and the conclusion follows directly from this observation.

Note that we may attain perfect recovery precisely when $A$ has full column rank, and a uniform upper bound on the pointwise error in reconstructing signals in $P C_{q}$ from (3.1) may be derived by Theorem 3.3. The following corollary makes use of the well-known fact that, given $D, E \in \mathbb{C}^{M \times M},\|D \otimes E\|_{\ell^{\infty}}=\|D\|_{\ell^{\infty}}\|E\|_{\ell^{\infty}}$, as induced matrix norms.

Corollary 3.4. Let $A \in \mathbb{C}^{N \times M}$ be with full column rank. Let $\epsilon>0$ and $q \in \mathbb{Z}_{+}$. Then, for $B \in \mathbb{C}^{N \times M}$ satisfying

$$
\left\|B^{\star} A-I_{M}\right\|_{\ell^{\infty}}<\sqrt[q]{\epsilon+1}-1
$$

we have $\left\|I-\Theta_{B}^{\star} \Theta_{A}\right\|_{P C_{q}}<\epsilon$.

Proof.

$$
\begin{aligned}
\left\|I-\Theta_{B}^{\star} \Theta_{A}\right\|_{P C_{q}} & =\left\|I_{M^{q}}-\left(\left(A^{\star} B\right)^{T}\right)^{\otimes q}\right\|_{\ell^{\infty}} \\
& =\left\|I_{M^{q}}-\left(I_{M}+\left(A^{\star} B-I_{M}\right)^{T}\right)^{\otimes q}\right\|_{\ell^{\infty}} \\
& \leq \sum_{k=1}^{q}\left(\begin{array}{l}
q \\
k
\end{array}\right)\left\|\left(A^{\star} B-I_{M}\right)^{T}\right\|_{\ell^{\infty}}^{k} \\
& =\left(\left\|\left(A^{\star} B-I_{M}\right)^{T}\right\|_{\ell^{\infty}}+1\right)^{q}-1 \\
& =\left(\left\|B^{\star} A-I_{M}\right\|_{\ell^{\infty}}+1\right)^{q}-1 \\
& <\epsilon .
\end{aligned}
$$

We note that this upper bound is a significant improvement of the one presented in [6].

\section{Operators on $L^{2}[0,1]$ satisfying Cuntz-like relations}

An important topic in signal analysis is the method by which we may decompose and then recover data. Only half of the relations in the Cuntz algebra setting suffice to perform this task, whereas the remaining Cuntz 
relations establish a splitting of the signal into orthogonal components. In this section we describe a procedure for constructing a finite collection of linear operators with the flexibility of partially or fully satisfying the Cuntz-like relations.

Let $A, B \in \mathbb{C}^{N \times M}$. Define the collection of filters $\left\{m_{j, A}\right\}_{j=0}^{N-1}$ corresponding to $A$ by

$$
m_{j, A}(x)=\sum_{k=0}^{M-1} A_{j, k} f_{k}(x),
$$

where $f_{k}(x)$ are either $\sqrt{M} \chi_{[k / M,(k+1) / M)}(x)$ or $e^{2 \pi i k x}$. We refer to the collection of filters as piecewise-type when $f_{k}$ are the characteristic functions and as exponential-type when $f_{k}$ are the complex exponential functions.

Depending on the context, there are advantages to using one type of filter over the other. For example, it is not too complicated to see that, in expressing $m_{j, C}$ in terms of $m_{j, A}$ and $m_{j, B}$, it is substantially simpler to use filters of piecewise-type for $C=A \oplus B$ and filters of exponential-type for $C=A \otimes B$.

Proposition 4.1. Let $A \in \mathbb{C}^{N_{1} \times M_{1}}$ and $B \in \mathbb{C}^{N_{2} \times M_{2}}$. For piecewise-type filter functions,

$$
m_{j, A \oplus B}(x)= \begin{cases}m_{j, A}\left(\frac{M_{1}+M_{2}}{M_{1}} x\right) & \text { if } 0 \leq j \leq N_{1}-1 ; \\ m_{j-N_{1}, B}\left(\frac{M_{1}+M_{2}}{M_{2}} x-\frac{M_{1}}{M_{2}}\right) & \text { if } N_{1} \leq j \leq N_{1}+N_{2}-1 .\end{cases}
$$

For exponential-type filter functions,

$$
m_{j, A \otimes B}(x)=m_{\left\lfloor j / N_{2}\right\rfloor, A}\left(M_{2} x\right) m_{\left(j \bmod N_{2}\right), B}(x) .
$$

Proof. The first identity is fairly clear to see, so we omit the proof. Instead, we show the second identity:

$$
\begin{aligned}
m_{j, A \otimes B}(x) & =\sum_{k=0}^{M_{1} M_{2}-1}(A \otimes B)_{j, k} e^{2 \pi i k x} \\
& =\sum_{l=0}^{M_{1}-1} \sum_{k=l M_{2}}^{(l+1) M_{2}-1}(A \otimes B)_{j, k} e^{2 \pi i k x} \\
& =\sum_{l=0}^{M_{1}-1} A_{\left\lfloor j / N_{2}\right\rfloor, l} \sum_{k=l M_{2}}^{(l+1) M_{2}-1} B_{\left(j \bmod N_{2}\right), k-l M_{2}} e^{2 \pi i k x} \\
& =\sum_{l=0}^{M_{1}-1} A_{\left\lfloor j / N_{2}\right\rfloor, l} e^{2 \pi i l M_{2} x} \sum_{k=0}^{M_{2}-1} B_{\left(j \bmod N_{2}\right), k} e^{2 \pi i k x}
\end{aligned}
$$

as desired.

Now let us define the operators $\left\{S_{j, A}\right\}_{j=0}^{N-1}$ corresponding to $A$ on $L^{2}[0,1]$ by

$$
S_{j, A} f(x)=m_{j, A}(x) f(r(x)),
$$

where $r(\omega)=M \omega \bmod 1$. A simple calculation provides the adjoint operator

$$
S_{j, A}^{\star} f(x)=\frac{1}{M} \sum_{\omega: r(\omega)=x} \overline{m_{j, A}(\omega) f} f(\omega) .
$$

The result that follows characterizes when these operators intermingle to satisfying some Cuntz-like relations. 
Theorem 4.2. Let $A, B \in \mathbb{C}^{N \times M}$. The following results hold when the filter functions are of piecewise-type or of exponential-type. The following conditions are equivalent:

$$
\begin{aligned}
& \text { (i) } S_{j, A}^{\star} S_{k, B}=\delta_{j, k} I \text { for all } j, k \in\{0,1, \ldots, N-1\} \\
& \text { (ii) } B A^{\star}=I_{N}
\end{aligned}
$$

Moreover, the conditions are also equivalent:

$$
\begin{aligned}
& \text { (iii) } \sum_{j=0}^{N-1} S_{j, B} S_{j, A}^{\star}=I \\
& \text { (iv) } A^{\star} B=I_{M}
\end{aligned}
$$

Proof. We will prove the theorem for the case of piecewise-type filter functions and defer the case of exponential-type to the next section, as it is more technical.

We ascertain the equivalence of conditions (i) and (ii) through the following calculation:

$$
\begin{aligned}
S_{j, A}^{\star} S_{k, B} f(x) & =\frac{1}{M} \sum_{\omega: r(\omega)=x} \overline{m_{j, A}(\omega)} m_{k, B}(\omega) f(r(\omega)) \\
& =f(x) \sum_{\omega: r(\omega)=x} \sum_{t=0}^{M-1} \overline{A_{j, t}} \chi_{[t / M,(t+1) / M)}(\omega) \sum_{s=0}^{M-1} B_{k, s} \chi_{[s / M,(s+1) / M)}(\omega) \\
& =f(x) \sum_{\omega: r(\omega)=x} \sum_{t=0}^{M-1} \overline{A_{j, t}} B_{k, t} \chi_{[t / M,(t+1) / M)}(\omega) \\
& =f(x)\left(B A^{\star}\right)_{k, j} .
\end{aligned}
$$

Now, suppose condition (iv) is satisfied.

$$
\begin{aligned}
\sum_{j=0}^{N-1} S_{j, B} S_{j, A}^{\star} f(x) & =\sum_{j=0}^{N-1} m_{j, B}(x) \frac{1}{M} \sum_{\omega: r(\omega)=r(x)} \overline{m_{j, A}(\omega) f} f(\omega) \\
& =\sum_{j=0}^{N-1} \sum_{s=0}^{M-1} B_{j, s} \chi_{[s / M,(s+1) / M)}(x) \sum_{\omega: r(\omega)=r(x)} \sum_{t=0}^{M-1} \overline{A_{j, t}} \chi_{[t / M,(t+1) / M)}(\omega) f(\omega) \\
& =\sum_{s=0}^{M-1} \sum_{t=0}^{M-1} \sum_{j=0}^{N-1} B_{j, s} \overline{A_{j, t}} \sum_{\omega: r(\omega)=r(x)} \chi_{[s / M,(s+1) / M)}(x) \chi_{[t / M,(t+1) / M)}(\omega) f(\omega) \\
& =\sum_{s=0}^{M-1} \sum_{t=0}^{M-1}\left(A^{\star} B\right)_{t, s} \sum_{\omega: r(\omega)=r(x)} \chi_{[s / M,(s+1) / M)}(x) \chi_{[t / M,(t+1) / M)}(\omega) f(\omega) \\
& =\sum_{s=0}^{M-1} \sum_{\omega: r(\omega)=r(x)} \chi_{[s / M,(s+1) / M)}(x) \chi_{[s / M,(s+1) / M)}(\omega) f(\omega) \\
& =f(x) \sum_{s=0}^{M-1} \chi_{[s / M,(s+1) / M)}(x) \\
& =f(x) .
\end{aligned}
$$


This concludes that condition (iii) is valid. Conversely, suppose condition (iii) is satisfied. Fix an integer $0 \leq$ $k \leq M-1$, and consider the characteristic function supported on $[k / M,(k+1) / M)$,

$$
\begin{aligned}
\chi_{[k / M,(k+1) / M)}(x) & =\sum_{j=0}^{N-1} S_{j, B} S_{j, A}^{\star} \chi_{[k / M,(k+1) / M)}(x) \\
& =\sum_{j=0}^{N-1} m_{j, B}(x) \frac{1}{M} \sum_{\omega: r(\omega)=r(x)} \overline{m_{j, A}(\omega)} \chi_{[k / M,(k+1) / M)}(\omega) .
\end{aligned}
$$

Then, for $x \in[r / M,(r+1) / M)$, we have

$$
\delta_{k, r}=\sum_{j=0}^{N-1} B_{j, r} \overline{A_{j, k}}=\left(A^{\star} B\right)_{k, r},
$$

where $\delta_{k, r}$ is the Kronecker delta. This establishes that condition (iv) is valid.

Corollary 4.3. Let $A \in \mathbb{C}^{N \times N}$. The collection of operators $\left\{S_{j, A}\right\}_{j=0}^{N-1}$ generates the Cuntz algebra $\mathcal{O}_{N}$ if and only if $A$ is unitary.

\section{Operators on $\ell^{2}(\mathbb{Z})$ satisfying Cuntz-like relations}

In this section, inspired by Appendix C: A tale of two Hilbert spaces in [8], we describe a collection of operators on $\ell^{2}(\mathbb{Z})$ which are closely connected to the operators $S_{j, A}$ on $L^{2}[0,1]$ of the previous section. We then take advantage of the appealing feature that describing signals in subspaces of $\ell^{2}(\mathbb{Z})$ is much more manageable for discrete data. Finally, we show an example of an image signal decomposed in a manner similar to discrete wavelet transform, where the operators $S_{j, A}$ correspond to a matrix $A$ without a constant first row. In this case the Walsh system is not an ONB; however, the first half of the Cuntz relations allows decomposition and reconstruction of a signal. Throughout this section, we assume that $A, B \in \mathbb{C}^{N \times M}$ and that the filter functions $m_{j, A}$ are of exponential-type, i.e.,

$$
m_{j, A}(x)=\sum_{k=0}^{M-1} A_{j, k} e^{2 \pi i k x} .
$$

Let us define the operators $\left\{\tilde{S}_{j, A}\right\}_{j=0}^{N-1}$ corresponding to $A$ on $\ell^{2}(\mathbb{Z})$ by

$$
\tilde{S}_{j, A} v(n)=\sum_{k \in \mathbb{Z}} h_{j, A}(n-M k) v(k),
$$

where $h_{j, A} \in \ell^{2}(\mathbb{Z})$ is given by

$$
h_{j, A}(k)=\left\{\begin{aligned}
A_{j, k} & \text { if } k \in\{0,1, \ldots, M-1\} \\
0 & \text { otherwise }
\end{aligned}\right.
$$

A simple calculation provides the adjoint operator

$$
\tilde{S}_{j, A}^{\star} v(n)=\sum_{k \in \mathbb{Z}} \overline{h_{j, A}(k-M n)} v(k) .
$$

The diagram below illustrates an intertwining feature of the operators $S_{j, A}$ and $\tilde{S}_{j, A}$. More precisely, the diagram commutes, that is $\tilde{S}_{j, A} \circ \theta=\theta \circ S_{j, A}$, where $\theta: L^{2}[0,1] \rightarrow \ell^{2}(\mathbb{Z})$ is the canonical isometric isomorphism given by

$$
\theta f(n)=\left\langle f, e^{2 \pi i n x}\right\rangle
$$


Thus $\theta$ is unitary, and its adjoint is

$$
\theta^{\star} v=\sum_{k \in \mathbb{Z}} v(k) e^{2 \pi i k x}
$$

Then, to convert between the operators on $L^{2}[0,1]$ and the operators on $\ell^{2}(\mathbb{Z})$, we have the conjugation tranformations: $S_{j, A}=\theta^{\star} \tilde{S}_{j, A} \theta$ and $\tilde{S}_{j, A}=\theta S_{j, A} \theta^{\star}$.

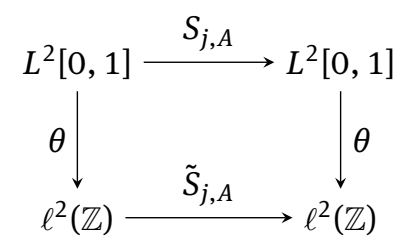

Figure 2: Diagram of the Intertwining of $S_{j, A}$ and $\tilde{S}_{j, A}$.

Theorem 5.1. Let $A \in \mathbb{C}^{N \times M}$. The operator (5.1) intertwines the operators $S_{j, A}$ and $\tilde{S}_{j, A}$, that is $\tilde{S}_{j, A} \circ \theta=\theta \circ S_{j, A}$, for all $j \in\{0,1, \ldots, N-1\}$.

Proof. It is sufficient to show $\tilde{S}_{j, A} \theta\left(e^{2 \pi i n x}\right)=\theta S_{j, A}\left(e^{2 \pi i n x}\right)$ for all $n \in \mathbb{Z}$. We first evaluate the left hand-side at the index $m \in \mathbb{Z}$ :

$$
\begin{aligned}
\tilde{S}_{j, A} \theta\left(e^{2 \pi i n x}\right)(m) & =h_{j, A}(m-M n) \\
& =\left\{\begin{aligned}
A_{j, m-M n} & \text { if } m-M n \in\{0,1, \ldots, M-1\} ; \\
0 & \text { otherwise }
\end{aligned}\right.
\end{aligned}
$$

We next evaluate the right-hand side at the index $m \in \mathbb{Z}$. We begin by expanding $S_{j, A}\left(e^{2 \pi i n x}\right)$,

$$
\begin{aligned}
S_{j, A}\left(e^{2 \pi i n x}\right) & =\sum_{k=0}^{M-1} A_{j, k} e^{2 \pi i k x} e^{2 \pi i n r(x)} \\
& =\sum_{k=0}^{M-1} A_{j, k} e^{2 \pi i(k+n M) x} .
\end{aligned}
$$

Then we have

$$
\begin{aligned}
\theta S_{j}\left(e^{2 \pi i n x}\right)(m) & =\left\langle S_{j}\left(e^{2 \pi i n x}\right), e^{2 \pi i m x}\right\rangle \\
& =\sum_{k=0}^{M-1} A_{j, k}\left\langle e^{2 \pi i(k+M n) x}, e^{2 \pi i m x}\right\rangle \\
& =\left\{\begin{aligned}
A_{j, m-N n} & \text { if } m-M n \in\{0,1, . ., M-1\} \\
0 & \text { else }
\end{aligned}\right.
\end{aligned}
$$

as desired.

We now proceed towards reproducing Theorem 4.2 for the operators $\left\{S_{j, A}\right\}$ on $L^{2}[0,1]$ generated by the exponential-type filter functions. This is done by working with the associated operators $\left\{\tilde{S}_{j, A}\right\}$ in the intertwining of Theorem 5.1 through the matrix representation of the operators

$$
\begin{aligned}
& \tilde{S}_{j, A}^{\star} \tilde{S}_{k, B} \text { for } j, k \in\{0,1, \ldots, N-1\}, \\
& \sum_{k=0}^{N-1} \tilde{S}_{k, B} \tilde{S}_{k, A}^{\star} .
\end{aligned}
$$


In what follows, we denote the canonical ONB of $\ell^{2}(\mathbb{Z})$ by the collection $\left\{e_{n}\right\}_{n \in \mathbb{Z}}$, i.e., $e_{n}(m)=\delta_{m, n}$. Recall that the matrix representation of an operator $T: \ell^{2}(\mathbb{Z}) \rightarrow \ell^{2}(\mathbb{Z})$ is the bi-infinite matrix whose $(n, m)$-entry is $T\left(e_{m}\right)(n)$. For convenience, we denote the matrix representation of the identity operator on $\ell^{2}(\mathbb{Z})$ by $I_{\infty}$. The matrix representations of the operators above are fairly simple to derive, so we state the following proposition without proof.

Proposition 5.2. Let $A, B \in \mathbb{C}^{N \times M}$. The matrix representations of (5.2) and (5.3) are the block diagonal matrices $\left(B A^{\star}\right)_{k, j} I_{\infty}$ and $I_{\infty} \otimes\left(A^{\star} B\right)^{T}$, respectively.

The following corollary is an immediate consequence of Theorem 5.1 and Proposition 5.2.

Corollary 5.3. Let $A, B \in \mathbb{C}^{N \times M}$. Then $S_{j, A}^{\star} S_{k, B}=\left(B A^{\star}\right)_{k, j} I$.

Before proceeding, we first take a short digression to make the action of the matrix representation $I_{\infty} \otimes\left(A^{\star} B\right)^{T}$ on $v \in \ell^{2}(\mathbb{Z})$ explicit. Letting $v_{n}=(v(M n), v(M n+1), \ldots, v(M(n+1)-1))$, we observe that $\left(I_{\infty} \otimes\left(A^{\star} B\right)^{T}\right) v=\oplus_{n \in \mathbb{Z}}\left(A^{\star} B\right)^{T} v_{n}$. Then, by considering $I_{\infty} \otimes\left(A^{\star} B\right)^{T}$ as acting on orthogonal subspaces, we have the following result.

Lemma 5.4. Let $C \in \mathbb{C}^{M \times M}$. Then $\left\|I_{\infty} \otimes C\right\|_{\ell^{2}(\mathbb{Z})}=\|C\|_{\ell^{2}\left(\mathbb{C}^{M}\right)}$.

Now we may determine exactly how close the operator (5.3) is to the identity operator with respect to the operator norm.

Proposition 5.5. Let $A, B \in \mathbb{C}^{N \times M}$. Then

$$
\left\|I_{\infty}-\sum_{k=0}^{N-1} \tilde{S}_{k, B} \tilde{S}_{k, A}^{\star}\right\|_{\ell^{2}(\mathbb{Z})}=\left\|I_{M}-A^{\star} B\right\|_{\ell^{2}\left(\mathbb{C}^{M}\right)} .
$$

Proof. From Proposition 5.2 and Lemma 5.4, we have

$$
\begin{aligned}
\left\|I_{\infty}-\sum_{k=0}^{N-1} \tilde{S}_{k, B} \tilde{S}_{k, A}^{\star}\right\|_{\ell^{2}(\mathbb{Z})} & =\left\|I_{\infty}-I_{\infty} \otimes\left(A^{\star} B\right)^{T}\right\|_{\ell^{2}(\mathbb{Z})} \\
& =\left\|I_{\infty} \otimes\left(I_{M}-\left(A^{\star} B\right)^{T}\right)\right\|_{\ell^{2}(\mathbb{Z})} \\
& =\left\|I_{M}-\left(A^{\star} B\right)^{T}\right\|_{\ell^{2}\left(\mathbb{C}^{M}\right)} \\
& =\left\|I_{M}-A^{\star} B\right\|_{\ell^{2}\left(\mathbb{C}^{M}\right)} \cdot
\end{aligned}
$$

Corollary 5.6. Let $A, B \in \mathbb{C}^{N \times M}$. Then

$$
\left\|I-\sum_{k=0}^{N-1} S_{k, B} S_{k, A}^{\star}\right\|_{L^{2}[0,1]}=\left\|I_{M}-A^{\star} B\right\|_{\ell^{2}\left(\mathbb{C}^{M}\right)} .
$$


Proof. To condense the notation, let $\Psi$ and $\psi$ be the operators

$$
\begin{aligned}
& \Psi=I-\sum_{k=0}^{N-1} S_{k, B} S_{k, A}^{*}, \\
& \psi=I_{\infty}-\sum_{k=0}^{N-1} \tilde{S}_{k, B} \tilde{S}_{k, A}^{\star} .
\end{aligned}
$$

By Theorem 5.1, we have $\Psi=\theta^{\star} \psi \theta$. Since $\theta$ is unitary, Proposition 5.5 implies that

$$
\begin{aligned}
\|\Psi\|_{L^{2}[0,1]} & =\sup _{\|f\|_{L^{2}}=1}\|\Psi f\|_{L^{2}[0,1]} \\
& =\sup _{\|\theta f\|_{\ell^{2}}}\|\psi \theta f\|_{\ell^{2}(\mathbb{Z})} \\
& =\|\psi\|_{\ell^{2}(\mathbb{Z})} \\
& =\left\|I_{M}-A^{\star} B\right\|_{\ell^{2}\left(\mathbb{C}^{M}\right)} .
\end{aligned}
$$

Altogether, Corollary 5.3 and Corollary 5.6 complete the proof of Theorem 4.2 for the operators $S_{j, A}$ and $S_{j, B}$ defined by the exponential-type filters. This was accomplished by showing (Theorem 5.2 and Proposition 5.5) that the associated operators $\tilde{S}_{j, A}$ and $\tilde{S}_{j, B}$ intermingle to mimic the Cuntz-like relations, so we have those additional results.

In this section we develop an application of the operators $\left\{\tilde{S}_{j}\right\}_{j=0}^{N-1}$ to discrete data. For each $q \in \mathbb{Z}_{+}$, we denote by $\tilde{\mathcal{H}}_{q}$ the subspace of $\ell^{2}(\mathbb{Z})$ given by $\tilde{\mathcal{H}}_{q}=\operatorname{span}\left\{e_{k} \mid k=0,1, \ldots, M^{q}-1\right\}$. The matrix representation of the linear operator $\left.\tilde{S}_{j}\right|_{\tilde{\mathcal{H}}_{q}}$ is the $M^{q+1} \times M^{q}$ matrix

$$
\left(\begin{array}{cccc}
A_{j, 0} & 0 & \ldots & 0 \\
\vdots & \vdots & \ddots & \vdots \\
A_{j, M-1} & 0 & \ldots & 0 \\
0 & A_{j, 0} & \ldots & 0 \\
\vdots & \vdots & \ddots & \vdots \\
0 & A_{j, M-1} & \ldots & 0 \\
\vdots & \vdots & \ddots & \vdots \\
0 & 0 & \ldots & A_{j, 0} \\
\vdots & \vdots & \ddots & \vdots \\
0 & 0 & \ldots & A_{j, M-1}
\end{array}\right) .
$$

Since the range of $\left.\tilde{S}_{j}\right|_{\tilde{\mathcal{H}}_{q}}$ is embedded in $\tilde{\mathcal{H}}_{q+1}$, we may view its matrix representation as inflating the dimension of data by a factor of $M$. The matrix representation of the adjoint operator $\left(\left.\tilde{S}_{j}\right|_{\tilde{\mathcal{H}}_{q}}\right)^{\star}=\left.\tilde{S}_{j}^{\star}\right|_{\tilde{\mathcal{H}}_{q+1}}$ is the adjoint of the matrix above. Here, since the range of $\left.\tilde{S}_{j}^{\star}\right|_{\tilde{\mathcal{H}}_{q+1}}$ is embedded in $\tilde{\mathcal{H}}_{q}$, we may view its matrix representation as deflating the dimension of the data by a factor of $M$.

Example 5.7. In the classic case $N=2$ there are two filters, lowpass and highpass, however for $N \geq 3$ this is not necessarily maintained anymore. If $A$ is a $3 \times 3$ unitary matrix having a constant $1 / \sqrt{3}$ row, then the filter corresponding to it can be interpreted as lowpass but there are intermediate ones whose action on the image may give negative values. We are still able to 'see' the action of the transform and the multiresolutions through a normalization of the values of the transformed signal. The pictures in the figure above represent first and second iterates on columns followed by rows of the image signal $\mathrm{f}$ according to the cascade algorithm, as seen here: 

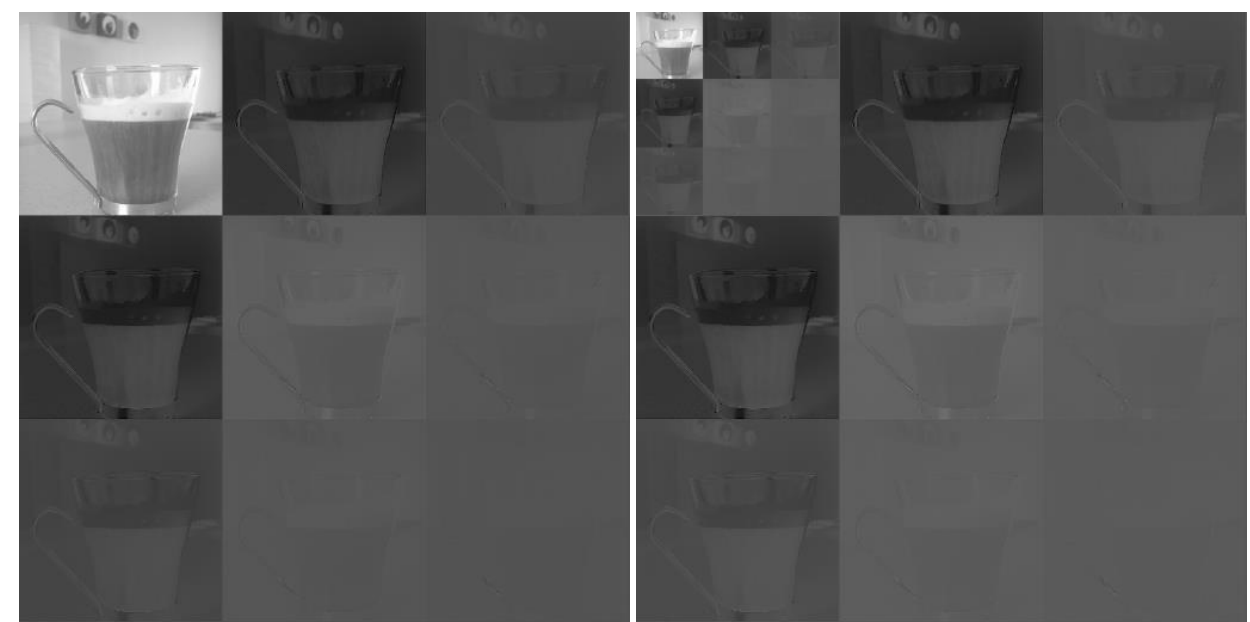

(a) Transform $A$, First Iterate

(b) Transform $A$, Second Iterate

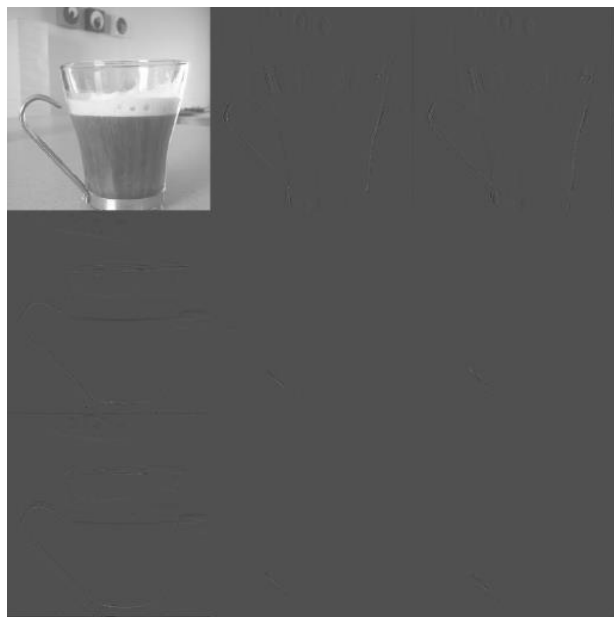

(c) Transform $B$, First Iterate

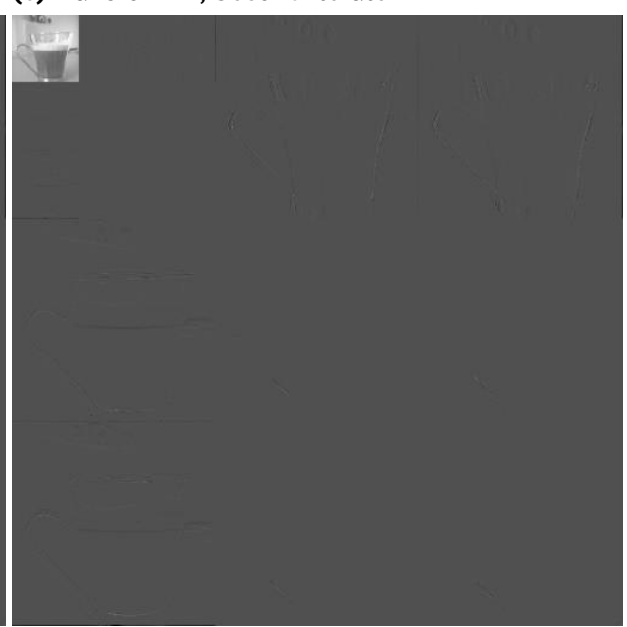

(d) Transform $B$, Second Iterate

Figure 3: Generalized discrete wavelet transform for matrices $A$ and $B$.

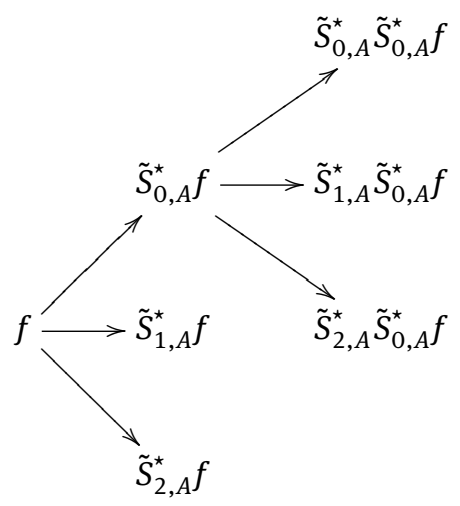

The wavelet-like transform was implemented using the following matrices:

$$
A=\left(\begin{array}{ccc}
1 / 3 & 1 / 2 & (1 / 6) \sqrt{23} \\
(-1 / 9) \sqrt{23} \sqrt{3} & 0 & (2 / 9) \sqrt{3} \\
(1 / 9) \sqrt{3} & (-1 / 2) \sqrt{3} & (1 / 18) \sqrt{23} \sqrt{3}
\end{array}\right), \quad B=\left(\begin{array}{ccc}
1 / \sqrt{3} & 1 / \sqrt{3} & 1 / \sqrt{3} \\
-.2 & -.5855 & .7855 \\
-.7916 & .5690 & .2226
\end{array}\right)
$$


Notice both matrices are unitary ( $B$ 's entries were truncated) but matrix $A$ does not satisfy the constant row condition.

\section{References}

[1] Dutkay D. E., Picioroaga G., Song M. S., Orthonormal bases generated by Cuntz algebras, J. Math. Anal. Appl., 2014, 409(2), 1128-1139

[2] Walsh J. L., A closed set of normal orthogonal functions, Amer. J. Math, 1923, 45(1), 5-24

[3] Vilenkin N., On a class of complete orthonormal systems, Bull. Acad. Sci. Math. [lzv. Akad. Nauk SSSR Ser. Mat.], 1947, 11(4), 363-400

[4] Chrestenson H. E., A class of generalized Walsh functions, Pacic. J. Math.,1955, 5(1), 17-31

[5] Dutkay D. E., Picioroaga G., Silvestrov S., On generalized Walsh bases, Acta Appl. Math., 2018,1-18, https://doi.org/10. 1007/s10440-018-0214-x

[6] Harding S. N., Generalized Walsh transforms, Cuntz algebras representations and applications in signal processing, University of South Dakota, Master Thesis, 2015, Copyright - Database copyright ProQuest LLC

[7] Dutkay D. E., Picioroaga G., Generalized Walsh bases and applications, Acta Appl. Math., 2014, 133(1), 1-18, https://doi. org/10.1007/s10440-013-9856-x

[8] Jorgensen P. E. T., Analysis and Probability: Wavelets, Signals, Fractals, Springer-Verlag New York, 2006 